\title{
INTEGRATED REMOTE SENSING AND GIS FOR MODELING ECONOMIC REHABILITATION DEVELOPMENT OF EX-MINE SITES
}

\author{
Junaidi and Eric K.H. Goh* \\ Amquest Research, PPKBSM, USM Engineering Campus, \\ Universiti Sains Malaysia, 14300 Nibong Tebal, Penang, Malaysia
}

Received 14 August 2006

\begin{abstract}
The potential environmental impacts of mining, increasing environmental legislation and public awareness have received increased attention world-wide in the last two decades. The focus of concern by the industry, environmental regulatory agencies and members of the public is the systematic rehabilitation of ex-mine sites to improve the quality at site for potential future commercial land use. The minerals extracted from these mine/quarry sites are essential in the construction, semiconductor, high-technology, ceramic and other manufacturing sectors for further industrial development. However, efficient engineering design and systematic economic evaluation of mine sites for site rehabilitation are required in maintaining the expected standards of environmental compliance. With escalating production costs and the keen competitiveness of the mining industry world-wide, the necessity to increase the efficiency in site rehabilitation is getting more prominence. A coordinated environmental protection and rehabilitation programme is essential if the environmental awareness of the community and the demands of the respective planning authorities are to be accommodated. There is thus a need to increase the base of knowledge for efficient planning in the systematic and progressive rehabilitation of current and future ex-mine sites. An efficient modeling tool is required for the systematic planning and design of potential economic land development of ex-mine sites. The applicability of Remote Sensing and Geographic Information System (GIS) technology is a useful tool to acquire spatial information for the systematic design and planning of potential development of ex-mine sites. This research was conducted to detect the trends in the suitability of land cover changes via land cover change detection of ex-mine sites and validated with reality. The findings are useful to assist in the development of a tool for efficient modeling and design of potential economic development of ex-mine sites. The aim of this research is to quantify, model and map the economic potential of the ex-mine sites for built up areas such as housing and other urban infrastructures. Land cover classes were interpreted into maps and the accuracy of the maps were validated to reference data and actual ground scenarios. The study for validation of the proposed modeling tool was carried out using the large prominent mining area in Malaysia namely the Kinta District. Results from the validation study carried out indicate that the correlation of the results obtained from this Integrated Remote Sensing and GIS tool for modeling to field data is in the range of $0.87-0.92$ which is acceptable and close to reality.
\end{abstract}

Keywords: minesite rehabilitation; remote sensing; GIS; economic land development potential

\footnotetext{
* Corresponding author e-mail: eric@eng.usm.my
} 


\section{INTRODUCTION}

An effective and economic rehabilitation design programme requires systematic planning from the outset, paying attention to the existing environment and in developing innovative techniques for the restoration of land after it has been disturbed. Effective planning and design of post-mine site rehabilitation has emerged as a significant engineering task due to the costs involved in phases for site rehabilitation and the regulatory requirements aimed at minimising off-site effects. Cost optimisation in site rehabilitation operations seeks to minimise the expenditure for overburden rehandling through systematic engineering design. Hoare [1] observed that in some instances rehabilitation costs can be as high as $\mathrm{A} \$ 100,000$ per hectare if additional environmental repairs are required for disturbed sites. There is thus an incentive to ensure that site rehabilitation works are done once and done well to avoid these excessive supplementary high costs. Engineering rehabilitation is concerned with the creation of suitable landforms compatible with the surrounding landscape whilst satisfying stability, drainage and other environmental requirements. The aim of efficient land management is the balance of productivity and costs to achieve optimum performance in the usage of any equipment for progressive rehabilitation of mine sites.

\section{CLASSIFICATION}

For efficient mine site management; extraction techniques and the post-mine usage of the land need to be identified early in the planning process. This is to allow for the extraction and subsequent rehabilitation phase to be integrated for increased efficiency. The following are suggested classification for the types of mine sites based on their types of mines, location and surrounding land use [2]:

- Type I: Undeveloped mine sites which are located in forest reserves and are not suited to surface disturbance through mine. Underground method may be more suitable for the extraction of the mineral at this site with minimum surface disturbance.

- Type II: This category encompasses operating mine sites where there is sufficient mineral mines and the opportunity to further expand the industry further through extending the area of the existing site lease.

- Type III: This class applies to those existing on-going mine sites where a constraint exists for future expansion of the surface mine lease due to legislative requirements or proximity to urban or residential areas.

- $\quad$ Type IV: This classification consists of old mine sites which are in an environment which does not permit further expansion or are already non-economical for further operations. The limitations for expansion could include the limits of the mine site lease being too close to residential or commercial areas. Recreational or housing development options could thus be integrated into the initial development plans on commencement of mine operations.

- Type $V$ : Mine sites which are remote or far away enough from existing urban or residential development sites could be planned for conversion into a potential commercial or industrial development site upon conclusion of operations.

\section{ECONOMIC POTENTIAL USES OF EX-MINE SITES}

The aim of any civic conscious mine site management staff is to restore the affected area to the land utilisation prior to mineral extraction, or to a better or improved land use. Post mine site usage should be established as early as possible in the planning phase to allow for the systematic progressive design of the site during mine production. The design of the proposed final 
landscape of the post mine site must also conform to the requirements of evolving legislation and community expectations. The proposed post mine land use should be compatible with adjacent land use and where applicable conform to existing local or state land use policies and future development plans.

The potential land use of ex-mine sites fall within the following categories:

- Wildlife/conservation parks,

- Industrial sites/parks,

- Residential/housing estates,

- Agriculture/livestock rearing,

- Aquaculture sites,

- Sports/recreational facilities,

- Water storage/management facilities,

- Education/afforestation research facilities, and

- Solid waste/landfill sites.

The potential of the ex-mine site to satisfy one of the above categories depends on the following factors:

- Economic, social and local factors of the proposed project,

- Land use pattern in the neighbouring areas adjacent to the mine site,

- Physical characteristics of the mine site and extraction technique,

- Whether the proposed land use is compatible with existing government policies and plans,

- Environmental impact to the adjacent sites due to the proposed change in land use,

- Potential change to the pattern of the local catchment area and water characteristics at site, and

- Expectations of local residents.

\section{METHODOLOGY}

\subsection{Integrated remote sensing and GIS technique}

Remote Sensing and Geographic Information System (GIS) play a critical role in the mapping process to acquire spatial information effectively for the update and rechecking of existing databases. Longley, Goodchild, Mcguire and Rhind [3] are of the opinion that a GIS-based data model can play an important role in voluminous global spatial data analysis where a vast and increasing array of geographical phenomena is represented in digital form. From literature reviews it was observed that the GIS method has demonstrated to being effective in exploring locational scenarios and visualizing spatial out-come. Data exploration within GIS is the domain of spatial data analysis technique. In this study, GIS-based techniques was conducted to detect ex-mine sites cover change trend for initial modeling of potential built up areas since results of land cover analysis will confirm with reality of actual land use (Fig. 1). The land cover maps are useful for delineating land use maps. The objective of this research is to assess the advantages of integrating remotely sensed data with GIS for land cover change detection. 


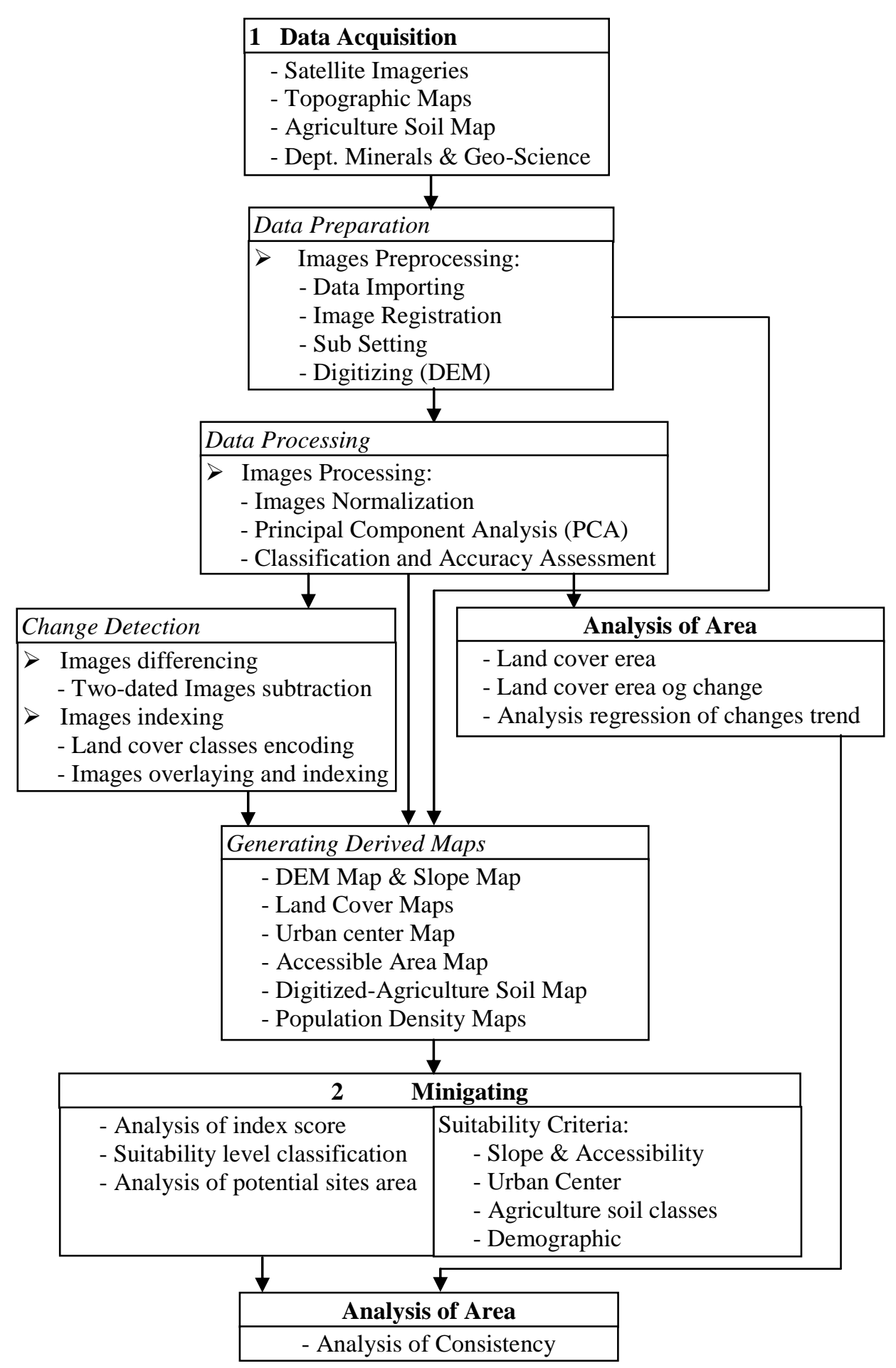

Fig: 1. Methodology of flowchart for rehabilitation project development. 


\subsection{Data types and acquisition}

Data used for modeling potential economic land development of ex-mine sites are categorized as primary and secondary data. Primary data comprise cartography data, satellite imageries and ground truth data. Secondary data consists of interviews, and other recorded data existing in the respective government databases. Geological maps and agricultural soil class data with the aim to improve accuracy of this modeling research project are used to supplement existing satellite images, topographic and land use maps. Interviews with relevant government departments should be conducted to compile any existing information of mining activities in the area under study. Information on present site environments should be collected via field visits. Additional information, such as census statistics, can be collected from the Internet and from the respective government statistical departments.

\subsection{Image pre-processing}

Before classification of any study area can be undertaken, there are several preprocessing steps to be carried out namely: data import, mosaicking, radiometric and geometric correction, rectification, sub- setting, digitizing [4], standardizing atmospheric condition [5], haze removal, and topographic normalization [6]. The data processing phase requires distinct data formatting to be applied in a subsequent process stage. As such, data conversion is required to transform a certain data format to another configuration [7]. Some of raster and vector data format commonly used in a digital image processing include: JPEG, Imagine file (img), generic binary, Spatial data transfer standard (SDTS), Arc View shape file(shp), Arc/Info Generate file and AutoCad Digital Exchange files (DXF) [4]. Mosaicking is the process of combining a set of separate images into a single seamless image. The input images must contain a map with the same projection [4].

\subsection{Image processing}

Data correction, however, is the improvement of image quality for subsequent data processing. Generally, there are two types of data correction namely radiometric and geometric correction. Changes in atmospheric conditions caused by pollutants in the environment might influence the characteristics of the signature reflectant and thus the accuracy of final interpretation. Application of radiometric enhancement is thus useful to avoid misinterpretation of the final results of change detection. A radiometric correction is a correction of digital numbers (DNs) or pixel intensity variations, which are not caused by the object being scanned, but it is due to differing sensitivity or malfunctioning of detectors, topographic effects, and atmospheric effect. Furthermore a radiometric correction involved images normalization, standardized atmospheric condition, and haze reduction/removal [4].

Image normalization, which covers one or more of several processes, is designed to bring multidate images data on a par such that a direct comparison of the gray values give an indication of actual or true change [8]. The aim is to minimize or remove the effects of different atmospheric (albedo variation) and topographic conditions. In practice, the images acquired from different dates appear different due to several reasons such variations in reflectance due to different sun elevation angles, change in atmospheric condition, variation in gain setting of various sensors and land cover changes. Therefore, it is necessary to normalize image radiometrically for subsequent applications. Among the most commonly used radiometric normalization techniques are pseudo-variat features, dark-pixel subtraction and relative radiometric normalization [9].

The normalized image was transformed on a pixel-by-pixel basis (overlay operation) using the following algorithm. 


$$
D N_{n o r-I I}=\left(s d_{i m-I}^{1 / 2} * s d_{i m-I I}^{-1 / 2}\right)\left(D N_{i m-I I}-M_{i m-I I}\right)+M_{i m-I}
$$

where:

$D N_{\text {nor-II }}=$ New normalized DN of image-II,

$D N_{i m-I I}=$ original DN of image-II, $s d_{i m-I}=$ standard deviation (dispersion matrix) of image-I,

$s d_{i m-I I}=$ standard deviation (dispersion matrix) of image-II,

$M_{\text {im-II }}=$ Mean vector of image-II, and

$M_{i m-I}=$ Mean vector of image-I.

Radiometric calibration and correction are applied to eliminate or to reduce the differences between images as result of changing atmospheric conditions [10]. Geometric correction addresses errors in the position of pixels. It is applied to raw sensor data to correct errors of perspective due to the earth's curvature (terrain variation) and sensor motion effects (sensor viewing geometry). A multi-date dataset analysis requires accurate geometric co-registration [11]. Since registration error could potentially be interpreted as land cover change, Milne proposed that the two images should be registered within the accuracy of one pixel or less to avoid misinterpretation of the changes [12]. This registration phase brings all data to the same scale and geometry. This method was undertaken to improve the quality of the image data.

Rectification is the process of projecting the data onto a plane and making it conform to a map projection system. A rectification process involves geo-referencing that assigns map coordinates to the image data. [4]. The degree to which the rectification process is successful depends to a large extent of the choice of ground control points (GCPs) and the geometric model [13]. The Landsat geometric model is used for Landsat imaging taking into account the error of its sensor and earth's curvature; while the first order of polynomial transformation (linear transformation) is better for data that are already projected onto a plane and has no distortion such an image produced by scanning or digitizing a paper map. Furthermore, the rectification process was performed to register and resample the image to a certain projection such as defined by the Universal Tranverse Mercator technique. This projection technique divides the world into 60 designated zones.

Radiometric, spatial and spectral enhancements are different phases of the image processing technique. Radiometric enhancement can be defined as an enhancement of pixel value either by contras stretching, histogram manipulation and brightness inversion. Spatial enhancement included filtering and resolution merge (image fusion). Principal Component Analysis, an essential component in spectral enhancement, is an image transformation technique that is used for a variety of purposes in remote sensing and Geographic Information Systems, including data compression and change analysis [8].

\subsection{Principal component analysis}

The Principal Component Analysis (PCA) technique is applied to all Landsat TM bands to allow the redundant data to be compressed into a much smaller number of bands with little loss of information. The PCA of images are generated using all bands available from the Landsat TM data. Combination of PC-1, PC-3, PC-7 of the images have been then coded in red, green and blue, respectively. This combination is applied to get the best signature separability of the images. The principal components obtained from this analysis are then used in the classification phase.

\subsection{Classification}

Both supervised and unsupervised remote sensing classification methodologies were utilized in 
the multi-step approach for this research to derive the spectral signature for the various land surface cover classes. A maximum-likelihood study is then conducted using all bands for each sub scene. The outline sample or training area generated for each surface class are then used to provide information to the classification program with typical examples of each kind of land cover to be used in the classification. The statistical parameters generated from the training area compares the digital numbers (DNs) of every pixel in the image with these statistical parameters. The pixel was assumed to belong to the same surface class as the training area if the DNs for a pixel lies within a known training area. The signature from the surface of the training area is then collected and analyzed to obtain the information on the number of bands to be used for the best signature separabiliy in the classification phase. Thresholding, using the probability image file, is performed to screen out misclassified pixels in order to improve the quality of the classification results. Since the results obtained are statistically broad and not necessarily representative of the spectral signature of the entire study area, unsupervised classification is then performed to classify the object signatures. The raster-classified classes are subsequently edited to improve the accuracy in simulating the land cover map.

\subsection{Accuracy assessment}

Individual accuracy assessments studies are carried on and evaluated for each classes extracted from satellite data obtained. The assessment is undertaken using the stratified random sampling approach. An error matrix is developed supplemented by application of Kappa statistics which assesses the accuracy by incorporating the individual error of omission and commission. The results of accuracy calculation were divided into two types: namely producer accuracy and user accuracy. Producer accuracy is based on modeling, and user accuracy is referred to assessment based on the experience of the analyst. This assessment is based on the reference data that includes topographic maps and collected field data.

\subsection{Change detection}

The following image algebra of equation (2) is applied for change detection analysis [8]:

$$
\operatorname{Dijk}=B \operatorname{Vijk}(1)-B \operatorname{Vijk}(2)+c
$$

where:

Dijk = change pixel value,

$B \operatorname{Vijk}(1)=$ brightness value at time 1 ,

$B \operatorname{Vijk}(2)=$ brightness value at time 2 ,

$c=$ constant,

$i=$ line number,

$j=$ column number,

$k=$ a single band.

Further analyses of a certain object change to others from multi-date images are required to apply image data indexing. This is due to the limitation of the analytical software to cope with the large script required to produce change detection 'from-to' interpretation for several classes of multi-date images correctly. The weighting factor is given to this recoded data to avoid a redundant result. Figure 2 is the corresponding flowchart for change detection analysis. The image algebra of equation (3) is then performed for further analysis:

$$
C i j k=B R V i j(1) \pm B R V i j(2) \pm B R V i j(3) \pm B R V i j(n)
$$


where:

$\operatorname{BRVij}(\mathrm{n})=\mathrm{DN}_{\mathrm{ijk}}(\mathrm{n}) * \mathrm{WIF}$,

Cijk $=$ change pixel index value,

$\operatorname{BRVijk}(1)=$ brightness recoded value at time 1 ,

$B R V i j k(2)=$ brightness recoded value at time 2 ,

$\operatorname{BRVijk}(n)=$ brightness recoded value at time $\mathrm{n}$,

$D N=$ digital recoded number,

$W I F=$ weighting index factor (e.g., 1, 9 and etc.),

$i=$ line number, $\mathrm{j}=$ column number and $\mathrm{k}=$ single layer.

Analysis of changes trend involves the extrapolation technique and is based on the assumption that factors that influence the changes trend have an equal effect and continues for the future. This analysis is carried out with the assumption that the urban associated area, year and housing demand are considered independent variables.

\subsubsection{Spatial sitting analysis}

The land cover, slope, accessibility, center of development, agriculture soil, and urban demographic classes have been included in the spatial analysis to identify the potential uses of ex-mine sites with the aim to create an accurate GIS based data model. This analysis phase is carried out based on how all these spatial criteria are correlated in the spatial data analysis process. The algorithm recommended for spatial sitting analysis in the mitigating the economic potential of ex-mine sites are as follows [14]:

$$
\left.S I=\sum_{i}^{N 2}\left[R I W_{i}^{2} \cdot \sum_{j}^{N 3 i}\left(R I W_{i j}^{3}\right) R I W_{i j, k}^{4}\right)\right]
$$

where:

$S I=$ suitability index;

$N 2=$ the number of level 2 decision factor;

$R I W^{2}=$ the relative importance weight of level 2 decision factor I;

$N 3 \mathrm{i}=$ The number of level 3 sub factors directly connected to level 2 decision factor I;

$R I W^{3}{ }_{j}=$ the relative importance weight of level 3 sub factors $\mathrm{j}$ of level 2 decision factor I; and

$R I W_{j k}^{4}=$ the relative weight of level 4 attribute category $\mathrm{k}$ of level 3 sub factor $\mathrm{j}$ and level 2 decision factor $\mathrm{i}$.

Since this research focuses on the potential development of ex-mine sites, land cover of barren mining area was selected for the input in the spatial analysis model. Demographic analysis was performed to evaluate the possible economic land demand corresponding with increasing population growth.

\subsubsection{Modeling the suitability potential of ex-mine sites}

The potential area of change is modeled by incorporating the six inputs of spatial parameters or criteria. Potential economic land development of ex-mine sites must meet the following criteria: 
1. The area of potential site should be $10800 \mathrm{~m}^{2}$ or larger.

2. The site must be easily accessible.

3. The slope of the area must be less than $25 \%$.

4. The site must not be in any potential agricultural soil area.

5. The site must be near to the center of development.

6. The site must be in a relatively high population density area.

To identify which area is potential or suitable, the Suitability Index Method is applied to this model which is obtained using equation 3, under Change Detection. Since the analysis focuses on the mitigating potential built-up area that is derived from ex-mine sites, the GIS analysis model developed is based on the assumption that ex-mine sites cover area is a key factor. Giving the weighting value to each spatial effect generates the level of potential development for exmine sites. The weighting value of 3 applies to slopes with hazardous terrain. The weighting of 2 signifies urban center aesthetics and the level of population density (demographic factor). Assessing the score of the output pixel value for the new image generates the levels of development potential for the site under investigation.

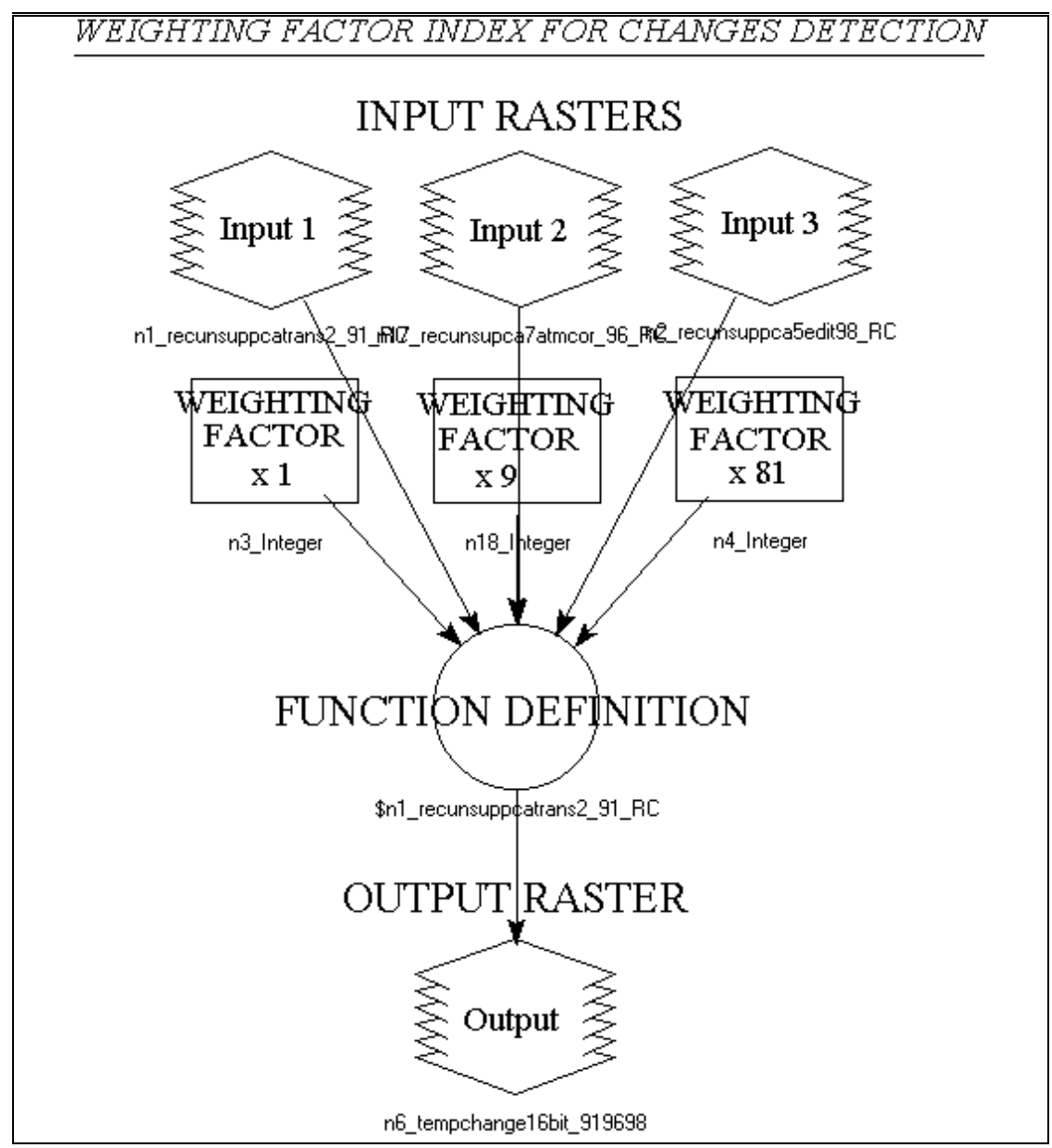

Fig. 2: Index Change Detection flowchart. 


\section{CASE STUDY - RESULTS AND DISCUSSION}

The aim of this research is to assess the benefits of using integrated Remote Sensing satellite images and Geographical Information System (GIS) for modeling and design of potential economic land development for ex-mine sites. This study is carried out for the detection and modeling of land use changes of ex-mine sites with the objective of determining the optimum economic usage of ex-mine sites for different site scenarios. The study area selected for this research is the mineral-rich area of Kinta, Malaysia. The Kinta District is located between $4^{0} 20^{\prime}$ and $4^{0} 45^{\prime} \mathrm{N}$ latitude and $100^{\circ} 50^{\prime}$ and $101^{\circ} 15^{\prime} \mathrm{E}$ longitude (Fig. 3). One of the most prominent features of the Kinta District site is the prominence of limestone hills. The main mountain ranges consist of extensive masses of granite where the original sedimentary cover has been removed by weathering and erosion. In addition, the characteristic features of the climate for the Kinta District site are uniform temperatures throughout the year $\left(36^{0} \mathrm{~F}-99^{0} \mathrm{~F}\right)$, high humidity (28 - $83 \%$ ), complemented by abundant rainfall in the annual range of 81 inches - 146 inches. The Kinta case study site is divided into two major distinct geologic zones namely Devonian sedimentary and metamorphic rock types. This study area was selected because:

a) The Kinta District is a major mining area contributing to national development and the nation's industrialisation programme.

b) The ex-mine sites in the Kinta District have been rapidly developing in the last decade by successfully evolving into residential, commercial and industrial areas.

c) The ready availability of spatial and non-spatial data is suitable for validation in this economic land development modeling research study for ex-mine sites.

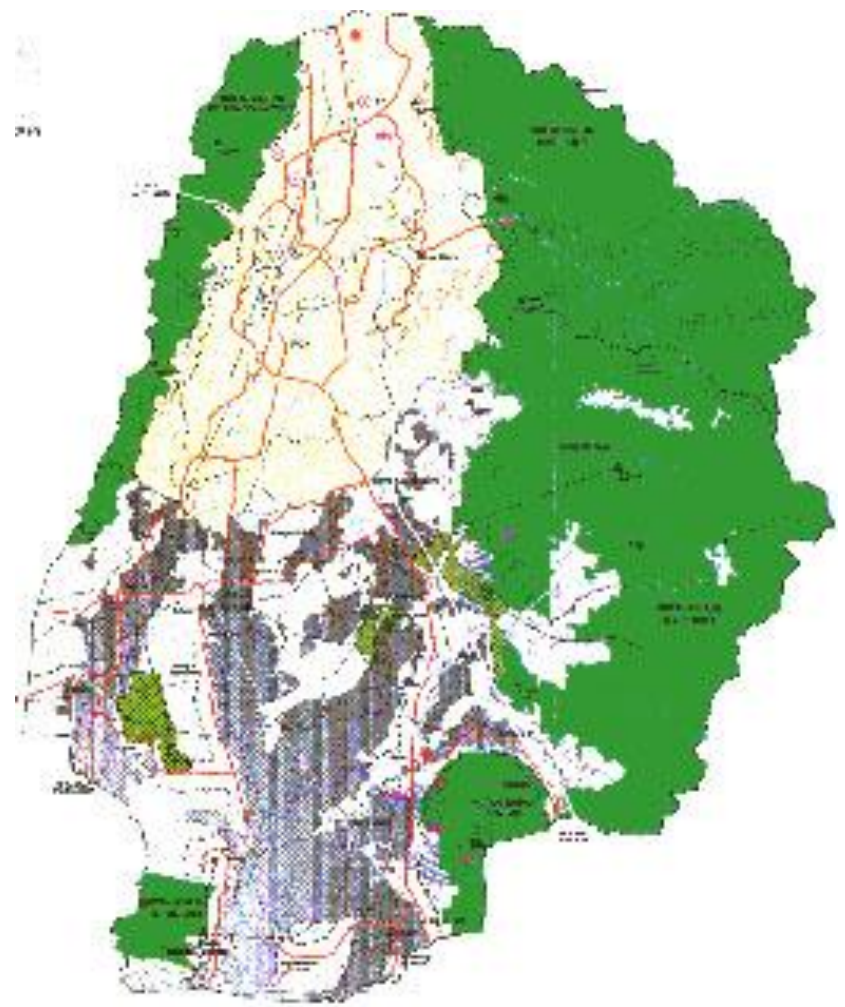

Fig. 3: Map of Study Area - Kinta District. 
Data used in this research are categorized as primary and secondary data. Primary data comprise cartography data, satellite imageries and ground truth data. Secondary data consists of interviews, census statistical report and other supported data from literature reviews. Cartography data such topographic maps were used to create a base map of the Kinta District whereas satellite imageries provide information on the multi-date land cover changes in the study area. The Landsat-TM digital satellite images of 1991, 1996 and 1998, topographic and land use maps data used in this study were supplemented by geological maps and agricultural soil class data. Site interviews were also carried out with relevant government departments to collect information on the scope of mining activities and the proposed aim of the respective administrative agencies planning for future development of the Kinta District. Site data was also collected from field visits. Other data used to support this research consists of information from the Internet such as statistical analysis and census statistical reports produced by the Department of Statistics (Malaysia). The respective digital images obtained from Landsat were geometrically corrected and subsequently geo-rectified accordingly. To avoid misinterpretation of object (land cover) all the images obtained must be registered within accuracy of one pixel or less. In this study, care has been specially taken to keep the root mean square error to be less than one.

The preprocessing phase involves image registration and normalization. Image registration is a crucial step carried out to ensure that the multi temporal data has the same scale and geometry. Results from the registration are based on images of the Kinta District which were geo-rectified to Universal Transverse Mercator (UTM) Clarke 1866 Zone 47 Standards. In this georectification process, the Landsat TM image of 1991 was used, whereas the topography map of 1991 was used as a reference. The Landsat TM images of 1996 and 1998 were also georectified, using the image to image rectified method. This pixel correction phase is carried out so that all the digital images of the Kinta District collected for 1996, 1998 correspond appropriately to that of 1991 with the aim that all data is brought to the same scale and geometry for ease of analysis in subsequent phases of research. Based on the accuracy assessment performed using the image processing software, Imagine, complemented with topographic maps and the ground data; the results show that the accuracy obtained from this exercise is reasonable. Accuracy of the assessment was based on the 1991, 1996 and 1998 data compiled.

The Principal Component Analysis phase is subsequently carried out on all Landsat-TM bands to obtain the best signature separation for the images for use in the classification phase. In order to enhance the quality in the classification of object signatures, the threshold technique was used to screen out misclassified pixels and the clustering routine applied to generate 20 spectral clusters. The clusters obtained are identified as variations of seven major land cover classes for the 1991 and 1996 satellite digital data sets; whereas the 1998 data set consists of six major land covers types. Individual accuracy assessments, using the stratified random sampling approach, were then carried out for each land cover class extracted from the satellite data obtained. 210 sample points were taken to improve the accuracy of the analysis.

The Digital Elevation Model (DEM) map was then generated by on screen digitizing of contour line of mosaiced topographic map scale 1: 50 000. An annotation layer was created and converted to img-format file for further GIS analysis. The non-linear rubber sheeting method was applied to generate 30 by 30 meter square grid. This method is used to produce a more natural contour from sparse data. The slope map was also derived from the 30 meter DEM and converted to degree unit. Three slope classes were designated for this study namely: 0 - 10 degree (gently slopes), 11 - 25 degree (moderate to steep slopes) and > 25 degree (very steep slopes). Land cover map is obtained as results of the images classification phase for the 1991, 1996 and 1998 Landsat images. Eight major land cover classes were identified namely: barren mining sites, urban associated sites, forest, agricultural, grass and sparse vegetation, water, cloud and shadowed area (outliers). A buffer zone was assigned in the creation of the Urban Center map. The buffer zone of $3.75 \mathrm{~km}$ was applied to Ipoh and $2.5 \mathrm{~km}$ for the other sites. 
These buffers were created based on the availability of infrastructures and public services in the respective area. An Accessible Area Map was created based upon the existence of a developed transportation network in the area. The buffer zone of $500 \mathrm{~m}, 500-5000 \mathrm{~m}$ and greater than 5000 were generated with the assumption that people can access the road respectively within the following designated time frame: less than 5 minutes, less than 30 minutes and greater than 30 minutes with a speed velocity of $10 \mathrm{~km} / \mathrm{h}$. The speed assumption was taken into consideration by observing the condition of the secondary roads in the respective mine sites. The Agriculture Soil Map obtained from the Kinta District Structure Planning Masterplan was then georectified with the topographic map of 1991. The annotation layer was created to classify the 5 divisions of suitability towards agriculture application namely: Class 1 (good soil for agricultural area with no conditional limitation), Class 2 (good soil for agricultural area with conditional limitation), Class 3 (moderate soil for it with at least 1 serious conditional limitation); and lastly Classes 4 and 5 (poor soils for agricultural area with serious and very serious conditional limitation).

Demographic analysis carried out in this research to evaluate the possible land demand dealing with corresponding population growth. To correlate the interaction of population demand on a land parcel, the 1980, 1991 and 2000 census data acquired from Department of Statistic (Malaysia) was used to estimate the housing demand in the near future. This demand value was applied to calculate the requirement of land parcel in the Kinta District. Housing demand was analyzed base on the new dwelling unit required due to projected population growth.

Results obtained using the GIS model for the suitability of potential economic land development of ex-mine sites shows that the range of ex-mine site index value ranges from 30 to 50 . The value ranging from 30 to 36 signifies low suitability and the total area obtained for this class for the Kinta District case study is $2.022 \mathrm{~km}^{2}$. The moderate suitability area ranges from 37 to 43 with a total area amounting to $49.85 \mathrm{~km}^{2}$. The index value having a high suitability area for economic land development ranges from 44 to 50 . Thus from this research the total area of high economic land development of ex-mine sites obtained for the Kinta District amounts to a total area of $81.53 \mathrm{~km}^{2}$.

Model validation is carried out by evaluating the consistency, precision and accuracy of information obtained. In this study, to generate the potential sites area two phases of validation have been performed; namely the land cover accuracy assessment and consistency analysis of the validated map. The accuracy was carry out by determining the correlation of the calculated to actual field data. A GIS validation model was used to check the consistency of the results obtained in this study. Validation of the proposed model was conducted using the GIS spatial overlaying model, which involved spatial potential sites of 1991 and spatial reality of 1991. The land cover map of 1998 was assumed to be the spatial reality of 1991 . The reality of low, moderate or high is interpreted as the replacement of each potential level of ex-mine sites to urban associated area in 1998. Results of the GIS validation model shows a ratio of $31.72 \%$, $19.67 \%$ and $16.60 \%$ of high-suitability, moderate-suitability and low-suitability ex-mine sites respectively have been converted into urban-associated areas within the period of seven years. The results show the consistency of the proposed model that is indicated by the highest change of the most potential sites of ex-mine sites and then followed by the lower classes. Information on validation of such interpretation, location, attributes and area of validated sites is as shown in Fig. 4. The results of this cross validation show that the model is consistent and applicable for future planning of potential economic development of ex-mine sites. Studies carried out specifically at the Sungai Raia site, indicates that the accuracy of this economic ex-mine land planning model developed reaches a high accuracy correlation of $85.66 \%$ with actual field data. The terrain models were subsequently obtained from overlaying of raster images on the digital elevation model (DEM) that has been analyzed. This model provides a visualization of the earth surface condition. 


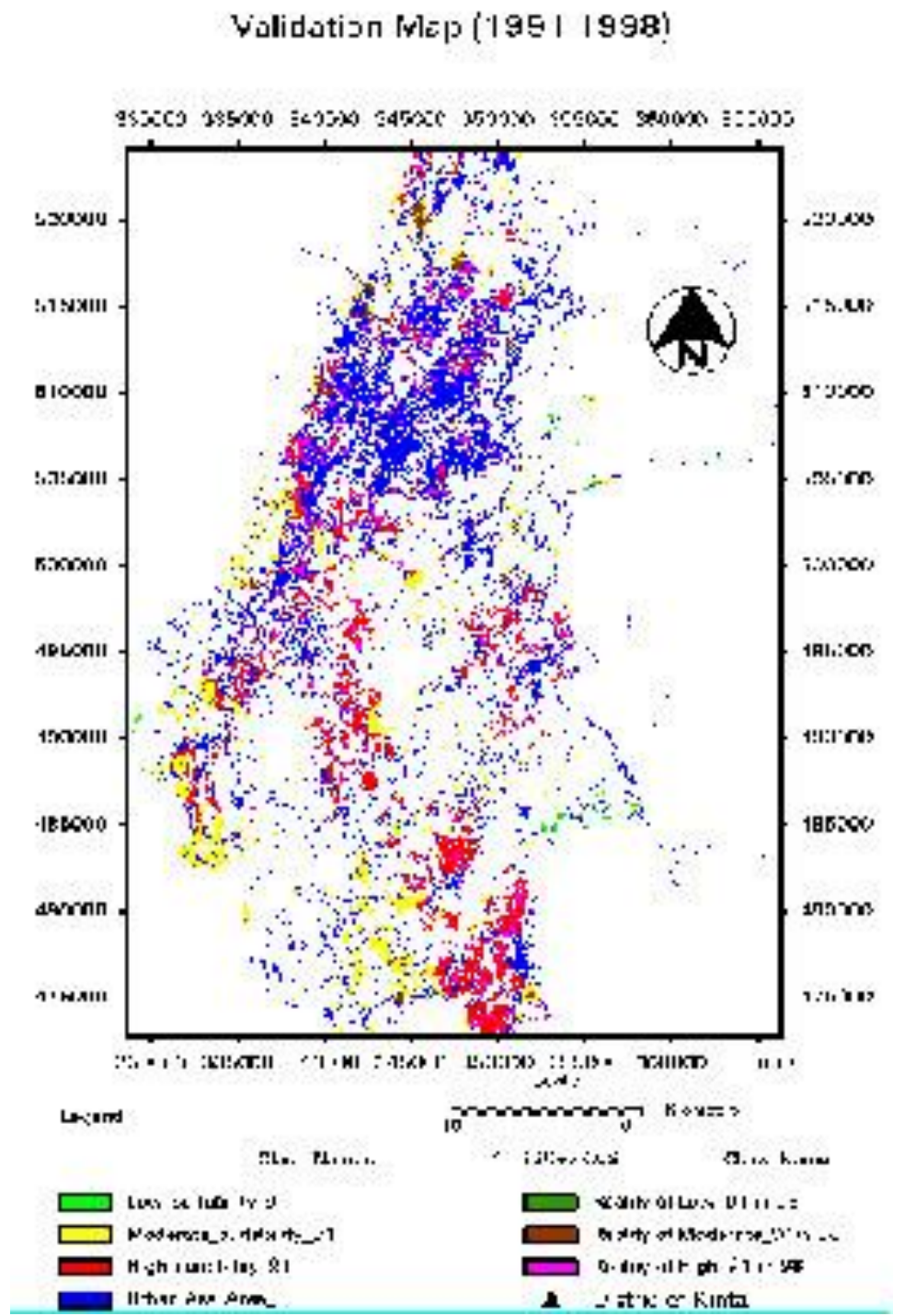

Fig. 4: Land Use Zoning Validation on suitability of ex-mine sites towards economic land development.

\section{CONCLUSION}

The encouraging results obtained from this research on the modeling of potential economic rehabilitation and planning of ex-mine sites for systematic national development indicates that:

- The integration of Geographic Information System (GIS) and Remotely Sensing Data can be a powerful tool for a computer-based land cover mapping exercise where remotely sensing data can be utilized for updating existing data, especially for ex-mine sites.

- Remote Sensing and Geographic Information system (GIS) technology can be used successfully to identify and quantify the land cover classes in any area under investigation as evidenced from the success in the Kinta District case study. In this study, remotely sensed data of 1991, 1996 and 1998 were acquired to generate six land cover classes with 
reasonable accuracy. In this research the accuracy obtained for the proposed model is high namely: $92.38 \%$ for $1991 ; 90.95 \%$ for 1996 and $87.62 \%$ for 1998 .

- Research results show that a combination of the Post-classification Method and Image Indexing Method has confirmed the development of a better technique in the identification of multi-temporal changes. Test results indicate the possibility to input more than two images in a single process with interpretable results.

- The GIS spatial model provides an enhanced environment for the analysis, evaluation and decision-making in preliminary mine site selection studies and the potential rehabilitation of the site after cessation of mineral extraction.

- Results obtained from these cross-validation studies indicate that the model developed is consistent and can confidently be applied for future land development planning of potential ex-mine sites.

\section{REFERENCES}

1. Hoare, R.G. (1985), The cost factor in rehabilitation choices, in Seminar Proceedings on coal mining as a land use, Australian Coal Association: Brisbane, pp. 204-212.

2. Robertson, A. (1998), Rehabilitation objectives and post mining land uses, Resource extractive Management, Resource extractive Managers Journal Ltd.: Nottingham, pp. 18-22.

3. Longley, P.A., Goodchild, M.F., Maguire, D.J., and Rhind, D.W. (1999), Geographical Information Systems. Second Edition, Vol. 1. New York: John Wiley and Sons, Inc, pp. 7-15.

4. ERDAS (1997), ERDAS Field Guides. Atlanta: ERDAS Inc, pp. 5-15.

5. Mah, A. (2000), Urban Planning and monitoring change using ER Mapper. GIS Web. (www.gisdvelopment.net/aars/acrs/2000/ts12).

6. Colby, J.D. and Keating, P.L. (1998), Land Use Cover Classification Using Landsat TM Imagery in the tropical highlands: The Influence of anisotropic Reflectance. International Journal of Remote Sensing, 19(8), pp. 1479-1500.

7. Hohl, P. (1998), GIS Data Conversion: Strategies, Techniques, and Management. USA. Onword Press.

8. Jensen, J.R. (1996), Digital Change Detection, Introduction Digital Image Processing - A Remote Sensing Perspective, Second Edition. New Jersey: Prentice Hall, pp. 257-279.

9. Lunetta, R.S. and Elvidge, C.D. (1999), Remote Sensing Change Detection. Environmental Monitoring Methods and Applications. London: Taylor and Francis Ltd., pp. 1-9.

10. Chaves, P.S. and MacKinnon, D. (1994), Automatic Detection of Vegetation Change in the Southwestern United States Using Remotely Sensed Images. Photogrammetric Engineering and Remote Sensing, pp. 571-583.

11. Singh, A. (1986), Change Detection in Tropical Forest Environment of Northern India Using Landsat. In Remote Sensing and Tropical Land Management (Eden, M.J. and Parry, J.T.),. New York: John Willey and Sons, pp. 237-254.

12. Milne, A. (1988), Change Detection Analysis Using Landsat Imagery: A review of Methodology. Proceeding of IGARSS'88 Symposium, Edinburgh, Scotland, September 13-16. Paris ESA Publication Division, pp. 541-544.

13. Gibson, P.J. (2000), Introductory Remote Sensing: Principles and Concepts. London: Taylor Francis Group, pp. 19-25.

14. Siddiqui, M.Z, Everett, J.W., and Vieux, B.E. (1996), Landfill Sitting Using Geographic Information System: A Demonstration. International Journal of Environmental Engineering, pp. 515-522. 